\title{
Solutions of the Diophantine Equation $\boldsymbol{x}^{3}+\boldsymbol{y}^{3}=\boldsymbol{z}^{3}-d$
}

\author{
By V. L. Gardiner, R. B. Lazarus and P. R. Stein
}

1. Introduction. In 195j, at the suggestion of Professor L. J. Mordell, Miller and Woollett [1] investigated the integer solutions of the equation

$$
x^{3}+y^{3}+z^{3}=d
$$

for all integers $0<d \leqq 100$. These authors carried out a numerical search in the range

$$
|x| \leqq|y| \leqq|z| \leqq 3200
$$

with the help of the EDSAC computer at Cambridge University; their results are tabulated in [1].

Mordell's original interest in this equation centered on the case $d=3$; in particular, he wanted to know whether there existed solutions in addition to the known triples $x=y=z=1$ and $x=y=4, z=-5$. For the range they considered, Miller and Woollett showed that in fact no further solutions existed. As a result of their happy decision to extend the search to other values of $d$, they discovered several other interesting properties of equation (1.1). Perhaps the two most striking facts were the following:

(a) For $d=2$, all solutions in the range (1.2) belong to the family:

$$
-6 t^{2}, \quad-6 t^{3}+1, \quad 6 t^{3}+1 .
$$

(b) Over the range considered, equation (1.1) has no solutions for the values $d=30,33,39,42,52,74,75,84,87$.

With regard to (b), it should be remarked that, while it has long been known [2] that equation (1.1) has no solutions if $d$ is an integer of the form $9 m \pm 4$, there is no known reason for excluding any other integer (except, of course, $d=0$ ). One might be tempted to conjecture that all integers (except zero) not of the form $9 m \pm 4$ can be expressed as the sum of three cubes, minus signs allowed. (If this conjecture were true, it would solve the so-called "Easier Waring's Problem" for cubes [2], since it would then follow that all integers can be expressed as the sum of at most four cubes.) Miller and Woollett's results seemed to cast a certain doubt on the soundness of such a conjecture; as will be seen below, further numerical experimentation has served to make it unlikely that the conjecture is true.

2. The Present Calculation. In the fall of 1961, Professor S. Chowla suggested to one of us (P.R.S.) that it would be of interest to investigate the case $d=3$ for a much larger range of $(x, y, z)$ values. Early in 1963 this suggestion was taken up and a program was written for the Laboratory's I.B.M. STRETCH Computer to

Received November 13, 1963. Revised December 11, 1963. This paper was performed under the auspices of the United States Atomic Energy Commission at the Los Alamos Scientific Laboratory of The University of California, Los Alamos, New Mexico. 
search for solutions of equation (1.1) in the equivalent form:

$$
x^{3}+y^{3}=z^{3}-d \text {. }
$$

The range chosen was:

$$
\begin{aligned}
& 0 \leqq x \leqq y \leqq 2^{16}=65,536 \\
& 0<N \leqq 2^{16}, \quad N \equiv z-x \\
& 0<|d| \leqq 999
\end{aligned}
$$

It will be observed that this range excludes negative values of $x, y, z$, although $d$ may have either sign. The small number of solutions (217 such) thus omitted were calculated separately on the MANIAC II Computer.

3. Results. The actual solutions found are collected in a large table, a copy of which has been deposited in the UMT file. A limited number of copies have been retained by the authors for distribution to interested mathematicians. The table is divided into three parts; Table I and Table II cover, respectively, the ranges $-999 \leqq d \leqq-2$ and $2 \leqq d \leqq 999$. Only "primitive" solutions are tabulated; these are solutions in which $x, y, z$ have no common factor. All "derived" solutions (the terms go back to Miller and Woollett) can be recovered by multiplication: e.g., if $d, x, y, z$ is a primitive solution, the associated derived solutions are $d^{\prime}=m^{3} d, x^{\prime}=$ $m x, y^{\prime}=m y, z^{\prime}=m z, m=-1, \pm 2, \pm 3, \cdots$. Values of $|d|$ which are themselves cubes have been omitted from the present calculation. There is a large number of solutions for each such case, and it was felt that their inclusion would make the tables too long. The additional solutions mentioned in Section 2, those for which one or two members of the triple $(x, y, z)$ are negative, have been arbitrarily assigned to Table II (positive $d$ ). With these conventions, the total number of primitive solutions found is 1873 for negative $d$ and 2148 for positive $d$. Except for the cubes $|d|=1,8,27,64$ (which we do not list), our results are in exact agreement with those of Miller and Woollett over the range they considered.

Finally, we have included a third table (Table III), which serves as a summary of our results. With the cubes omitted, the column labelled " $d$ " lists all integers $2 \leqq d \leqq 999$ which are not of the form $9 m \pm 4$. For each such entry, the column labelled $N_{+}$indicates the number of primitive solutions of (2.1) with $d$ positive, while $N_{-}$gives the corresponding number of solutions for negative $d$. This table is reproduced in the present paper (Table A).

4. Discussion. (a) For the range considered, there are 70 values of $|d|$ for which there exist no solutions of equation (2.1). In Table A these are indicated by an asterisk. There are in addition 12 values of $|d|$ which have only derived solutions, viz:

$$
|d|=24,80,192,250,375,384,480,624,744,768,808,960 .
$$

52 of the 70 "excluded" integers are of the form $9 m \pm 3,13$ are of the form $9 m \pm 2$, 4 are of the form $9 m \pm 1$ and one $(|d|=180)$ is divisible by 9 .

One of the "excluded" values noted by Miller and Woollett has gone away, 
TABLE A

\begin{tabular}{|c|c|c|c|c|c|c|c|c|c|c|c|}
\hline$d$ & $N_{+}$ & $N_{-}$ & $d$ & $N_{+}$ & $N_{-}$ & $d$ & $N_{+}$ & $N_{-}$ & $d$ & $N_{+}$ & $N_{-}$ \\
\hline 2 & 23 & 0 & 90 & 9 & 10 & 174 & 2 & 3 & 260 & 6 & 4 \\
\hline 3 & 1 & 1 & 91 & 4 & 3 & 177 & 1 & 1 & 261 & 3 & 2 \\
\hline 6 & 4 & 1 & 92 & 9 & 2 & 178 & 1 & 1 & 262 & 3 & 0 \\
\hline 7 & 1 & 2 & 93 & 2 & 0 & 179 & 1 & 4 & 263 & 1 & 1 \\
\hline 9 & 2 & 1 & 96 & 0 & 1 & $180^{*}$ & 0 & 0 & 264 & 0 & 3 \\
\hline 10 & 3 & 1 & 97 & 5 & 4 & 181 & 11 & 8 & 267 & 2 & 1 \\
\hline 11 & 4 & 1 & 98 & 2 & 2 & 182 & 2 & 2 & 268 & 0 & 1 \\
\hline 12 & 0 & 1 & 99 & 7 & 11 & 183 & 3 & 6 & 269 & 4 & 3 \\
\hline 15 & 2 & 1 & 100 & 3 & 0 & 186 & 1 & 1 & 270 & 2 & 1 \\
\hline 16 & 1 & 0 & 101 & 4 & 2 & 187 & 1 & 2 & 271 & 5 & 2 \\
\hline 17 & 4 & 3 & 102 & 0 & 1 & 188 & 6 & 3 & 272 & 4 & 5 \\
\hline 18 & 3 & 4 & 105 & 1 & 0 & 189 & 4 & 2 & 273 & 1 & 3 \\
\hline 19 & 2 & 2 & 106 & 3 & 2 & 190 & 16 & $1 \overline{1}$ & 276 & 0 & 1 \\
\hline 20 & 4 & 4 & 107 & 1 & 2 & 191 & 2 & 2 & 277 & 5 & 0 \\
\hline 21 & 3 & 2 & 108 & 3 & 1 & 192 & 0 & 0 & 278 & 3 & 5 \\
\hline 24 & 0 & 0 & 109 & 7 & 5 & $195^{*}$ & 0 & 0 & 279 & 7 & 9 \\
\hline 25 & 2 & 2 & $110^{*}$ & 0 & 0 & 196 & 2 & 4 & 280 & 3 & 5 \\
\hline 26 & 1 & 4 & 111 & 2 & 2 & 197 & 6 & 6 & 281 & 7 & 4 \\
\hline 28 & 3 & $\begin{array}{l}7 \\
3\end{array}$ & $114^{*}$ & 0 & 0 & 198 & 4 & $\begin{array}{l}0 \\
2\end{array}$ & 282 & 0 & 1 \\
\hline 29 & 4 & 3 & 115 & 2 & 1 & 199 & 2 & 3 & 285 & 1 & 2 \\
\hline $30^{*}$ & 0 & 0 & 116 & 2 & 0 & 200 & 1 & 1 & 286 & 2 & 2 \\
\hline $33^{*}$ & 0 & 0 & 117 & 3 & 0 & 201 & 0 & 1 & 287 & 6 & 7 \\
\hline 34 & 5 & 5 & 118 & 6 & 10 & 204 & 1 & 1 & 288 & 3 & 8 \\
\hline 35 & 5 & 2 & 119 & 4 & 0 & 205 & 2 & 1 & 289 & 2 & 1 \\
\hline 36 & 3 & 3 & 120 & 0 & 2 & 206 & 1 & 2 & $290^{*}$ & 0 & 0 \\
\hline 37 & 2 & 1 & 123 & 2 & 0 & 207 & 4 & $\overline{5}$ & 291 & 1 & 0 \\
\hline 38 & 1 & 2 & 124 & 1 & 1 & 208 & 1 & 3 & 294 & 1 & 3 \\
\hline $39^{*}$ & 0 & 0 & 126 & 8 & 2 & 209 & 8 & 10 & 295 & 5 & 6 \\
\hline $42^{*}$ & 0 & 0 & 127 & 5 & 4 & 210 & 1 & 1 & 296 & 2 & 1 \\
\hline 43 & 4 & 4 & 128 & 13 & 3 & 213 & 1 & 1 & 297 & 1 & 2 \\
\hline 44 & 1 & 0 & 129 & 2 & 1 & 214 & 2 & 2 & 298 & 6 & 2 \\
\hline 45 & 1 & 3 & 132 & 1 & 0 & 215 & 4 & 1 & 299 & 1 & 2 \\
\hline 46 & 2 & 2 & 133 & 3 & 4 & 217 & 6 & 4 & 300 & 0 & 4 \\
\hline 47 & 2 & 2 & 134 & 4 & 5 & 218 & 4 & $\begin{array}{l}2 \\
2\end{array}$ & 303 & 1 & 1 \\
\hline 48 & 2 & 0 & 135 & 2 & 1 & 219 & 1 & 1 & 304 & 2 & 4 \\
\hline 51 & 0 & 1 & 136 & 0 & 1 & 222 & 0 & 1 & 305 & 3 & $\begin{array}{l}x \\
3\end{array}$ \\
\hline $52^{*}$ & 0 & 0 & 137 & 5 & 0 & 223 & 5 & 3 & 306 & 4 & 4 \\
\hline 53 & 3 & 2 & 138 & 1 & 0 & 224 & 2 & 3 & 307 & 11 & 11 \\
\hline 54 & 4 & 1 & 141 & 2 & 1 & 225 & 10 & 5 & 308 & 1 & 1 \\
\hline 55 & 8 & 6 & 142 & 1 & 0 & 226 & 2 & 5 & 309 & 3 & 2 \\
\hline 56 & 1 & 2 & $143^{*}$ & 0 & 0 & 227 & 0 & 1 & $312^{*}$ & 0 & 0 \\
\hline 57 & 2 & 8 & 144 & 4 & 2 & 228 & 1 & 0 & 313 & 2 & 0 \\
\hline 60 & 2 & 1 & 145 & 1 & 1 & $231^{*}$ & 0 & 0 & 314 & 12 & 3 \\
\hline 61 & 1 & 1 & 146 & 2 & 3 & 232 & 4 & 3 & 315 & 7 & 4 \\
\hline 62 & 5 & 5 & 147 & 1 & 1 & 233 & 2 & $\stackrel{\square}{2}$ & 316 & 9 & 4 \\
\hline 63 & 5 & 3 & 150 & 0 & 1 & 234 & 0 & 1 & 317 & 0 & 2 \\
\hline 65 & 4 & 4 & 151 & 2 & 2 & 235 & 2 & 4 & $318^{*}$ & 0 & 0 \\
\hline 66 & 1 & 0 & 152 & $\overline{2}$ & 1 & 236 & 2 & 1 & $321^{*}$ & 0 & 0 \\
\hline 69 & 1 & 3 & 153 & $\overline{9}$ & 5 & 237 & 0 & 2 & 322 & 5 & 2 \\
\hline 70 & 2 & 4 & 154 & 3 & 6 & 240 & 1 & 0 & 323 & 7 & 15 \\
\hline 71 & 9 & 4 & 155 & 5 & 13 & 241 & 0 & 1 & 324 & 2 & 3 \\
\hline 72 & 1 & 1 & $156^{*}$ & 0 & 0 & 242 & 5 & 3 & $\begin{array}{l}024 \\
325\end{array}$ & 3 & $\begin{array}{l}0 \\
2\end{array}$ \\
\hline 73 & 5 & 2 & 159 & 1 & 4 & 243 & 1 & 1 & 326 & 1 & 1 \\
\hline $74^{*}$ & 0 & 0 & 160 & 4 & 2 & 244 & 6 & 3 & 327 & 1 & 0 \\
\hline $75^{*}$ & 0 & 0 & 161 & 8 & 8 & 245 & 2 & 3 & 330 & 5 & 1 \\
\hline 78 & 1 & 1 & 162 & 3 & 0 & 246 & 2 & 4 & 331 & 4 & 0 \\
\hline 79 & 3 & 0 & 163 & 4 & 2 & 249 & 1 & 1 & 332 & 1 & 4 \\
\hline 80 & 0 & 0 & 164 & 4 & 0 & 250 & 0 & 0 & 333 & 4 & 6 \\
\hline 81 & 2 & 1 & $165^{*}$ & 0 & 0 & 251 & 9 & 7 & 334 & 1 & $\begin{array}{l}0 \\
2\end{array}$ \\
\hline 82 & 1 & 2 & 168 & 1 & 2 & 252 & 8 & 5 & 335 & 2 & 5 \\
\hline 83 & 12 & 4 & $\begin{array}{l}169 \\
\text { S }\end{array}$ & 4 & 0 & 253 & 7 & 4 & 336 & 0 & 2 \\
\hline $84^{*}$ & 0 & 0 & 170 & 3 & 5 & 254 & 1 & 0 & 339 & 2 & 3 \\
\hline 87 & 1 & 0 & 171 & 7 & 3 & 255 & 3 & 1 & 340 & 1 & 0 \\
\hline 88 & 3 & 2 & 172 & 0 & 1 & 258 & 3 & 0 & 341 & 6 & 3 \\
\hline 89 & 0 & 3 & 173 & 1 & 0 & 259 & 3 & 5 & 342 & 6 & 15 \\
\hline
\end{tabular}


TABle A-Continued

\begin{tabular}{|c|c|c|c|c|c|c|c|c|c|c|c|}
\hline$d$ & $N_{+}$ & $N_{-}$ & $d$ & $N_{+}$ & $N_{-}$ & $d$ & $N_{+}$ & $N_{-}$ & $d$ & $N_{+}$ & $N_{-}$ \\
\hline 344 & 3 & 2 & 429 & 0 & 2 & 513 & 2 & 1 & 596 & 4 & 1 \\
\hline 345 & 1 & 0 & 430 & 2 & 2 & 514 & 1 & 6 & 597 & 0 & 1 \\
\hline 348 & 0 & 2 & 431 & 1 & 2 & 515 & 2 & 2 & $600^{*}$ & 0 & 0 \\
\hline 349 & 4 & 6 & 432 & 1 & 2 & $516^{*}$ & 0 & 0 & 601 & 8 & 4 \\
\hline 350 & 4 & 5 & 433 & 5 & 12 & 519 & 6 & 4 & 602 & 4 & 6 \\
\hline 351 & 4 & 2 & 434 & 9 & 3 & 520 & 2 & 0 & 603 & 13 & 6 \\
\hline 352 & 2 & 1 & $435^{*}$ & 0 & 0 & 521 & 7 & 8 & 604 & 6 & 4 \\
\hline 353 & 2 & 0 & 438 & 1 & 1 & 522 & 1 & 1 & 605 & 1 & 2 \\
\hline 354 & 1 & 0 & $439 *$ & 0 & 0 & 523 & 2 & 3 & $606^{*}$ & 0 & 0 \\
\hline 357 & 2 & 1 & 440 & 1 & 1 & 524 & 8 & 2 & $609^{*}$ & 0 & 0 \\
\hline 358 & 1 & 6 & 441 & 10 & 3 & 525 & 2 & 2 & 610 & 6 & 5 \\
\hline 359 & 5 & 3 & 442 & 6 & 3 & 528 & 0 & 1 & 611 & 0 & 3 \\
\hline 360 & 3 & 1 & 443 & 1 & 0 & 529 & 1 & 0 & 612 & 2 & 4 \\
\hline 361 & 3 & 2 & $444^{*}$ & 0 & 0 & $530^{*}$ & 0 & 0 & 613 & 3 & 2 \\
\hline 362 & 1 & 1 & 447 & 4 & 2 & 531 & 8 & 10 & 614 & 0 & 1 \\
\hline 363 & 1 & 1 & 448 & 1 & 3 & 532 & 10 & 10 & 615 & 4 & 1 \\
\hline $366^{*}$ & 0 & 0 & 449 & 11 & 9 & 533 & 1 & 5 & $618^{*}$ & 0 & 0 \\
\hline $367^{*}$ & 0 & 0 & 450 & 2 & 3 & $534^{*}$ & 0 & 0 & 619 & 1 & 2 \\
\hline 368 & 1 & 2 & 451 & 1 & 2 & 537 & 0 & 2 & 620 & 2 & $\overline{1}$ \\
\hline 369 & 5 & 3 & $452^{*}$ & 0 & 0 & 538 & 6 & 1 & 621 & 2 & 0 \\
\hline 370 & 3 & 5 & 453 & 0 & 1 & 539 & 6 & 4 & 622 & 5 & 2 \\
\hline 371 & 2 & 0 & 456 & 2 & 2 & 540 & 5 & 7 & 623 & 6 & 4 \\
\hline 372 & 2 & 3 & 457 & 2 & 2 & 541 & 8 & 5 & 624 & 0 & 0 \\
\hline 375 & 0 & 0 & 458 & 2 & 1 & $542^{*}$ & 0 & 0 & $627^{*}$ & 0 & 0 \\
\hline 376 & 1 & 0 & 459 & 2 & 2 & 543 & 1 & 1 & 628 & 0 & 2 \\
\hline 377 & 4 & 2 & 460 & 3 & 1 & 546 & 1 & 1 & 629 & 12 & 9 \\
\hline 378 & 7 & 3 & 461 & 5 & 6 & 547 & 3 & 7 & 630 & 7 & 1 \\
\hline 379 & 11 & 7 & $462^{*}$ & 0 & 0 & 548 & 0 & 3 & 631 & 6 & 6 \\
\hline 380 & 3 & 0 & 465 & 0 & 3 & 549 & 3 & 1 & 632 & 1 & 3 \\
\hline 381 & 1 & 3 & 466 & 1 & 1 & 550 & 2 & 1 & $633^{*}$ & 0 & 0 \\
\hline 384 & 0 & 0 & 467 & 6 & 12 & 551 & 3 & 2 & 636 & 1 & 0 \\
\hline 385 & 5 & 1 & 468 & 6 & 3 & 552 & 0 & 1 & 637 & 2 & 2 \\
\hline 386 & 5 & 1 & 469 & 6 & 8 & 555 & 0 & 1 & 638 & 11 & 13 \\
\hline 387 & 5 & 4 & 470 & 4 & 2 & $556^{*}$ & 0 & 0 & 639 & 5 & 5 \\
\hline 388 & 4 & 3 & 471 & 1 & 0 & 557 & 3 & 3 & 640 & 1 & 3 \\
\hline 389 & 1 & 1 & 474 & 1 & 1 & 558 & 8 & 5 & 641 & 1 & 0 \\
\hline $390^{*}$ & 0 & 0 & 475 & 2 & 9 & 559 & 6 & 6 & 642 & 1 & 0 \\
\hline 393 & 1 & 2 & 476 & 5 & 7 & 560 & 6 & 4 & 645 & 4 & 3 \\
\hline 394 & 0 & 1 & 477 & 5 & 3 & 561 & 2 & 2 & 646 & 2 & 0 \\
\hline 395 & 1 & 2 & $478^{*}$ & 0 & 0 & $564^{*}$ & 0 & 0 & 647 & 6 & 6 \\
\hline 396 & 2 & 4 & 479 & 1 & 1 & 565 & 0 & 1 & 648 & 1 & 2 \\
\hline 397 & 5 & 1 & 480 & 0 & 0 & 566 & 4 & 3 & 649 & 5 & 6 \\
\hline 398 & 5 & 8 & 483 & 1 & 0 & 567 & 4 & 4 & 650 & 2 & 0 \\
\hline 399 & 2 & 0 & 484 & 3 & 5 & 568 & 1 & 2 & 651 & 5 & 2 \\
\hline 402 & 0 & 1 & 485 & 1 & 1 & 569 & 2 & 2 & 654 & 1 & $\overline{1}$ \\
\hline 403 & 1 & 1 & 486 & 3 & 6 & 570 & 0 & 4 & 655 & 1 & 2 \\
\hline 404 & 2 & 1 & 487 & 6 & 2 & 573 & 1 & 1 & 656 & 4 & 3 \\
\hline 405 & 15 & 7 & 488 & 0 & 1 & 574 & 0 & 3 & 657 & 16 & 5 \\
\hline 406 & 6 & 2 & 489 & 2 & 5 & 575 & 7 & 8 & 658 & 8 & 5 \\
\hline 407 & 6 & 2 & 492 & $\overline{1}$ & 0 & 576 & 1 & 2 & 659 & 4 & 2 \\
\hline 408 & 1 & 0 & 493 & 2 & 3 & 577 & 8 & $\overline{1}$ & $660^{*}$ & 0 & 0 \\
\hline 411 & 1 & 2 & 494 & 1 & 3 & 578 & 1 & 2 & $663^{*}$ & 0 & 0 \\
\hline 412 & 1 & $\overline{2}$ & 495 & 7 & 4 & $579^{*}$ & 0 & 0 & 664 & 5 & 0 \\
\hline 413 & 8 & 2 & 496 & 5 & 6 & 582 & 2 & 1 & 665 & 7 & 10 \\
\hline 414 & 6 & 4 & 497 & 1 & 3 & 583 & 1 & 0 & 666 & 5 & 4 \\
\hline 415 & 4 & 1 & 498 & 2 & 1 & 584 & 0 & 3 & 667 & 3 & 4 \\
\hline 416 & 1 & 0 & $501 *$ & 0 & 0 & 585 & 1 & 1 & 668 & 2 & 2 \\
\hline 417 & 0 & 3 & 502 & 1 & 0 & 586 & 3 & 2 & 669 & 0 & $\overline{2}$ \\
\hline $420^{*}$ & 0 & 0 & 503 & 7 & 3 & 587 & 3 & 4 & 672 & 1 & 1 \\
\hline 421 & 6 & 8 & 504 & 1 & 1 & $588^{*}$ & 0 & 0 & 673 & 3 & 5 \\
\hline 422 & 1 & 0 & 505 & 5 & 8 & 591 & 2 & 0 & 674 & 4 & 3 \\
\hline 423 & 1 & 1 & 506 & 2 & 2 & 592 & 0 & 1 & 675 & 0 & 1 \\
\hline 424 & 3 & 2 & 507 & 1 & 2 & 593 & 4 & 1 & 676 & 1 & 0 \\
\hline 425 & 1 & 1 & 510 & 3 & 1 & 594 & 6 & 3 & 677 & 3 & 1 \\
\hline 426 & 2 & 0 & 511 & 7 & 4 & 595 & 2 & 2 & 678 & 2 & 5 \\
\hline
\end{tabular}


Table A-Continued

\begin{tabular}{|c|c|c|c|c|c|c|c|c|c|c|c|}
\hline$d$ & $N_{+}$ & $N_{-}$ & $d$ & $N_{+}$ & $N_{-}$ & $d$ & $N_{+}$ & $N_{-}$ & $d$ & $\Lambda_{+}$ & $N_{-}$ \\
\hline 681 & 1 & 0 & 765 & 0 & 1 & 848 & 7 & 1 & $933^{*}$ & 0 & 0 \\
\hline 682 & 2 & 1 & 766 & 5 & 4 & 849 & 2 & 0 & 934 & 0 & 1 \\
\hline 683 & 6 & 2 & $767^{*}$ & 0 & 0 & 852 & 0 & 1 & 935 & 7 & 2 \\
\hline 684 & 6 & 2 & 768 & 0 & 0 & 853 & 6 & 5 & 936 & 2 & 1 \\
\hline 685 & 8 & 5 & 771 & 4 & 2 & 854 & 6 & 2 & 937 & 10 & 7 \\
\hline 686 & 1 & 0 & 772 & 2 & 0 & 855 & 12 & 15 & 938 & 4 & 4 \\
\hline 687 & 2 & 4 & 773 & 0 & 4 & 856 & 2 & 5 & 939 & 0 & 2 \\
\hline 690 & 2 & 0 & 774 & 0 & 2 & 857 & 5 & 2 & 942 & 0 & 1 \\
\hline 691 & 1 & 1 & 775 & 1 & 5 & 858 & 1 & 0 & 943 & 3 & 6 \\
\hline 692 & 5 & 4 & 776 & 3 & 0 & $861^{*}$ & 0 & 0 & 944 & 1 & 5 \\
\hline 693 & 3 & 6 & $777^{*}$ & 0 & 0 & 862 & 3 & 7 & 945 & 2 & 2 \\
\hline 694 & 10 & 12 & 780 & 1 & 0 & 863 & 4 & 3 & 946 & 8 & 3 \\
\hline 695 & 1 & 1 & 781 & 2 & 2 & 864 & 3 & 1 & 947 & 2 & 1 \\
\hline 696 & 0 & 1 & 782 & 0 & 2 & 865 & 1 & 2 & $948^{*}$ & 0 & 0 \\
\hline 699 & 1 & 1 & 783 & 7 & 3 & 866 & 2 & 1 & 951 & 2 & 0 \\
\hline 700 & 2 & 2 & 784 & 0 & 2 & 867 & 4 & 3 & 952 & 2 & 0 \\
\hline 701 & 13 & 5 & 785 & 8 & 5 & $870^{*}$ & 0 & 0 & 953 & 5 & 5 \\
\hline 702 & 0 & 2 & $786^{*}$ & 0 & 0 & 871 & 0 & 1 & 954 & 2 & 3 \\
\hline 703 & 3 & 7 & $789^{*}$ & 0 & 0 & 872 & 6 & 2 & 955 & 2 & 0 \\
\hline 704 & 1 & 0 & 790 & 7 & 1 & 873 & 5 & 3 & 956 & 3 & 0 \\
\hline 705 & 0 & 1 & 791 & 8 & 1 & 874 & 12 & 11 & 957 & 4 & 1 \\
\hline 708 & 1 & 0 & 792 & 7 & 7 & 875 & 2 & 3 & 960 & 0 & 0 \\
\hline 709 & 2 & 0 & 793 & 2 & 1 & 876 & 2 & 3 & 961 & 4 & 1 \\
\hline 710 & 1 & 5 & 794 & 3 & 0 & 879 & 3 & 4 & 962 & 2 & 2 \\
\hline 711 & 1 & 2 & $795^{*}$ & 0 & 0 & 880 & 0 & 2 & 963 & 5 & 2 \\
\hline 712 & 3 & 0 & 798 & 1 & 5 & 881 & 10 & 12 & $964^{*}$ & 0 & 0 \\
\hline 713 & 4 & 3 & 799 & 2 & 3 & 882 & 3 & 7 & 965 & 5 & 4 \\
\hline 714 & 3 & 1 & 800 & 0 & 1 & 883 & 6 & 10 & 966 & 1 & 0 \\
\hline 717 & 1 & 0 & 801 & 5 & 4 & 884 & 0 & 1 & $969^{*}$ & 0 & 0 \\
\hline 718 & 0 & 1 & 802 & 3 & 1 & 885 & 2 & 3 & 970 & 2 & 1 \\
\hline 719 & 2 & 2 & 803 & 2 & 1 & 888 & 1 & 0 & 971 & 0 & 1 \\
\hline 720 & 7 & 6 & 804 & 3 & 2 & 889 & 1 & 1 & 972 & 4 & 3 \\
\hline 721 & 5 & 7 & 807 & 1 & 0 & 890 & 2 & 3 & 973 & 5 & 4 \\
\hline 722 & 2 & 9 & 808 & 0 & 0 & 891 & 3 & 0 & 974 & 1 & 7 \\
\hline 723 & 1 & 1 & 809 & 6 & 6 & 892 & 5 & 3 & $975^{*}$ & 0 & 0 \\
\hline 726 & 1 & 0 & 810 & 4 & 3 & 893 & 1 & 2 & 978 & 0 & 1 \\
\hline 727 & 4 & 4 & 811 & 11 & 12 & $894^{*}$ & 0 & 0 & 979 & 4 & 4 \\
\hline 728 & 5 & 1 & 812 & 1 & 2 & 897 & 0 & 1 & 980 & 5 & 5 \\
\hline 730 & 3 & 0 & 813 & 2 & 2 & 898 & 2 & 0 & 981 & 4 & 9 \\
\hline 731 & 1 & 2 & 816 & 1 & 0 & 899 & 6 & 6 & 982 & 1 & 0 \\
\hline $732^{*}$ & 0 & 0 & 817 & 1 & 0 & 900 & 1 & 2 & 983 & 3 & 2 \\
\hline $735^{*}$ & 0 & 0 & 818 & 8 & 14 & 901 & 4 & 2 & 984 & $\begin{array}{l}0 \\
3\end{array}$ & 0 \\
\hline 736 & 5 & 6 & 819 & 7 & 2 & 902 & 5 & 4 & 987 & $\begin{array}{l}0 \\
1\end{array}$ & 2 \\
\hline 737 & 1 & 2 & 820 & 13 & $1 \overline{3}$ & $903^{*}$ & 0 & 0 & 988 & 5 & 3 \\
\hline 738 & 2 & 0 & 821 & 1 & 2 & $906^{*}$ & 0 & 0 & 989 & 7 & 3 \\
\hline 739 & 3 & 2 & 822 & 1 & 0 & 907 & 2 & 2 & 990 & 3 & 4 \\
\hline 740 & 3 & 2 & 825 & 4 & 3 & 908 & 0 & 1 & 991 & 4 & 4 \\
\hline 741 & 2 & 0 & 826 & 1 & 0 & 909 & 5 & 7 & 992 & 0 & 1 \\
\hline 744 & 0 & 0 & 827 & 10 & 7 & 910 & 1 & 0 & 993 & 2 & 5 \\
\hline 745 & 1 & 1 & 828 & 4 & 2 & 911 & 13 & 8 & 996 & 0 & 1 \\
\hline 746 & 2 & 5 & 829 & 3 & 3 & $912^{*}$ & 0 & $\begin{array}{l}0 \\
0\end{array}$ & 997 & $\begin{array}{l}0 \\
3\end{array}$ & 1 \\
\hline 747 & 1 & 1 & $830^{*}$ & 0 & 0 & 915 & 0 & 1 & 998 & 4 & 1 \\
\hline 748 & 5 & 8 & 831 & 0 & 2 & 916 & 1 & 2 & 999 & 2 & 0 \\
\hline 749 & 2 & 4 & $834^{*}$ & 0 & 0 & 917 & 4 & 3 & & & \\
\hline 750 & 1 & 0 & 835 & 2 & 1 & 918 & $\begin{array}{l}x \\
10\end{array}$ & 5 & & & \\
\hline 753 & 1 & 0 & 836 & 0 & 1 & 919 & 6 & $\stackrel{0}{2}$ & & & \\
\hline $754^{*}$ & 0 & 0 & 837 & 4 & 5 & 920 & 0 & 1 & & & \\
\hline 755 & 17 & 11 & 838 & 3 & 1 & $921^{*}$ & 0 & 0 & & & \\
\hline 756 & 0 & 2 & 839 & 8 & 1 & 924 & 3 & 1 & & & \\
\hline 757 & 8 & 2 & 840 & 1 & 2 & 925 & 4 & 4 & & & \\
\hline $758^{*}$ & 0 & 0 & 843 & 0 & 1 & 926 & 3 & 2 & & & \\
\hline 759 & 0 & 1 & 844 & 1 & 2 & 927 & 1 & 3 & & & \\
\hline 762 & 2 & 0 & 845 & 0 & 2 & 928 & 2 & 1 & & & \\
\hline 763 & 0 & 5 & 846 & 4 & 7 & 929 & 0 & 1 & & & \\
\hline 764 & 5 & 4 & 847 & 6 & 2 & 930 & 0 & 3 & & & \\
\hline
\end{tabular}


namely $|d|=87$. The solution lies slightly beyond the range they considered:

$$
(1972)^{3}+(4126)^{3}=(4271)^{3}-87 .
$$

For $|d|=96$, Miller and Woollett found only one derived solution. There is actually a primitive solution for this case, but it lies well beyond their range:

$$
(10853)^{3}+(13139)^{3}=(15250)^{3}+96 .
$$

In general, it is rather risky to draw conclusions from the experimental evidence, even with a search as extensive as the present one. For example, $|d|=227$ and $|d|=971$ each have only a single solution, lying relatively close to the boundary of the search region:

$$
\begin{aligned}
(24579)^{3}+(51748)^{3} & =(53534)^{3}+227 \\
(7423)^{3}+(55643)^{3} & =(55687)^{3}+971 .
\end{aligned}
$$

Many other such examples can be found in our large table of solutions. Nevertheless, it is in our opinion rather unlikely that all the missing $|d|$ 's will turn out to be expressible as sums of three cubes. It would be of interest to attempt a proof that, say, 30 cannot be so expressed.

(b) All solutions for $|d|=2$ were found to belong to the parametric family (1.3). So far we have only succeeded in identifying one other family which is, in fact, a simple extension of (1.3). For $|d|=128$, all solutions are given by the formula:

$$
x=6 t^{2}, \quad y=-4+3 t^{3}, \quad z=4+3 t^{3}, \quad d=128 .
$$

If $t$ is even, the solutions are the derived ones associated with $d=2, m=4$, but for odd $t$ we get a new primitive family. (The existence of this parametric family was noted in [1].)

(c) The case $|d|=3$ was found to have no new solutions. $|d|=12$ may also be of theoretical interest; it is the smallest integer that appears to have only a single solution:

$$
7^{3}+10^{3}=11^{3}+12 .
$$

The next interesting case is $|d|=24$, which, in fact, has only the derived solutions:

$$
\begin{aligned}
(-2)^{3}+(-2)^{3} & =(2)^{3}-24 \\
8^{3}+8^{3} & =10^{3}+24 .
\end{aligned}
$$

Then, of course, comes $|d|=30$, the smallest integer for which no solution whatsoever has been found.

(d) As a final remark, we point out that our table affords an explicit decomposition into 4 or fewer cubes for every integer from 1 to 999 . In particular, every number of the form $9 m \pm 4$ in our range turns out to differ by a cube from a number for which one or more decompositions into 3 cubes has been found.

Los Alamos Scientific Laboratory

Los Alamos, New Mexico

1. J. C. P. Miller \& M. F. C. Woollett, "Solutions of the Diophantine equation $x^{3}+$ $y^{3}+z^{3}=k$," J. London Math. Soc., v. 30, 1955, p. 101-110.

2. G. H. HARDY \& E. M. WRIGHT, An Introduction to the Theory of Numbers, Third Edition, Clarendon Press, Oxford, 1954, p. 327. 